Proceedings of the 1996 IEEE

International Conference on Robotics and Automation

Minneapolis, Minnesota - April 1996

\title{
Adaptive Fuzzy Hybrid Force/Position Control for Robot Manipulators Following Contours of an Uncertain Object
}

\author{
Feng-Yih $\mathrm{Hsu}^{1}$ and Li-Chen $\mathrm{Fu}^{1,2}$ \\ Dept. of Electrical Engineering 1 \\ Dept. of Computer Science \& Information Engineering ${ }^{2}$ \\ National Taiwan University, Taipei, Taiwan, R.O.C.
}

\begin{abstract}
This paper proposes an adaptive fuzzy hybrid force/position control scheme, which can force the end effector of robot manipulators to follow the contour of an object in lack of knowledge of the exact geomeotric shape. The control objective is to perform hybrid force/position control regardless of the existence of the manipulator dynamics. The control algorithm proposed can adaptively update the position trajectory command as well as fuzzy control rules, and consequently, guarantee the global stability and drive the tracking errors to a neighborhood of zero. The present work is applied to the control of a five degreeof-freedom (DOF) articulated robot manipulator. Simulation results show that the proposed control architecture is featured in fast algorithmic convergence.
\end{abstract}

\section{Introduction}

A human, even if blind, can adapt to the uncertain surrounding environment by touching various objects there and gradually updating his perception about that world. But today's robot manipulators are lacking such kills, particularly, with respect to the varied contour-following tasks involving mechanic operations. The robot manipulators have to be taught or told in advance what the exact contour of object is so that they know how to perform the contour-following task. As a result, it will reduce flexible application of the robot manipulators when versatile tasks are designated to them. In fact, force sensing along the surface of an object can help the robot manipulators to recognize the uncertain environment, similar to human sensing. There have been several researches efforts related in estimation of constraint surface by force sensing [1]-[2].
Another occasion where the force sensing is applied to the robot manipulators is force/position control in performing contour-following tasks, for example, deburring tasks and grinding tasks. To achieve that control task, the robot end-effector has to follow the desired position trajectory as well as the desired force trajectory. Being different from the problem of sole position control, the above problem is not only to consider the robot manipulator dynamics but also to consider the interaction between the robot manipulators and the environment. Therefore, a force model to express the relation between the robot manipulators and the environment is necessary to analyze the problem. So far, the control schemes incorporating different kinds of force model may be classified into three types mainly: constraint robot control [3], compliant control [4]-[5] and hybrid force/position control[6]-[8]. For robot manipulators to perform contour-following tasks, the hybrid force/position control turns out to be a more suitable control approach since most object surfaces to be contacted are rigid enough, compared the passive devices mounted onto the end-effector, such as some elastic strings, so that transient excessive contact force can be avoided. In this paper, we focus our attention on the hybrid force/position control with uncertain shaped object.

On the other hand, the adaptive fuzzy control schemes have been demonstrated with better performance in the presence of system uncertainty [9]-[10]. Here, we will modify it to solve the problems mentioned above.

\section{Problem Formulation}

Consider a robot manipulator whose end-effector is in contact with an object with an aim to compliantly 


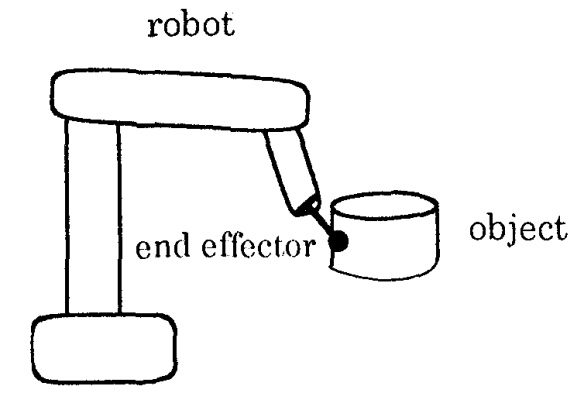

Figure 1: The robot performing contour-following

following a desired contour on the surface of the object, as depicted in Fig. 1. For simplicity in the coordinate transformation, we assume that the Cartesians coordinates of the end-effector in the world frame $\{W\}, x_{c}$ may be represented as a function of its joint coordinates in the reference frame, $q$, i.e.,

$$
x_{c}=H(q),
$$

where $x_{c}=\left[x_{c 1}, \cdots, x_{c 6}\right]^{T}=\left[x_{p}^{T}, x_{m}^{T}\right]^{T}$ with $x_{p} \in \Re^{3}$ being the position vector and $x_{m} \in \Re^{3}$ being the orientation vector, and $q=\left[q_{1}, \cdots, q_{n}\right]^{T}$. Differentiating equation (1), we can derive

$$
\dot{x}_{c}=\frac{\partial H(q)}{\partial q} \dot{q}=J(q) \dot{q},
$$

where $J(q) \in \Re^{6 \times n}$ is a Jocabin transform matrix and is assumed to be of full rank for $q$ lying in a compact set in the joint space, so that there exists a one-toone mapping between $x_{c}$ and $q$ in a properly defined compact set [11]. Thus, $J$ has a sudo-inverse matrix $J^{+}$, satisfying $J J^{+}=I$. Then, we can derive the dynamics of the robot manipulator in the world frame as follows:

$M\left(x_{c}\right) \ddot{x}_{c}+C\left(x_{c}, \dot{x}_{c}\right) \dot{x}_{c}+G\left(x_{c}\right)+D\left(x_{c}, \dot{x}_{c}\right)=F_{c}+F$,

where $M\left(x_{c}\right) \in \Re^{6 \times 6}$ is the inertia matrix, $C\left(x_{c}, \dot{x}_{c}\right) \dot{x}_{c}$ is the vector representing the centrifugal and Coriolis forces satisfying $\dot{M}-2 C$ is a skew-symmetric matrix, $G\left(x_{c}\right)$ is the vector of gravitational forces, $D\left(x_{c}, \dot{x}_{c}\right)$ is the vector of friction forces, $F_{c}$ is the vector consisting of contact forces and moments, and $F$ is the vector consisting of control input forces and moments. Also, the torque vector $\tau$ in the joint coordinate can be derived as $\tau=J^{T}(q) F$.

Given the robot dynamics (3), more importantly, the control task is to perform the force control as well as the position control. Therefore, in addition to the dynamics terms of robot manipulators, the contact force term $F_{c}$ in (3) has to be compensated, particularly, in the force control problem, leading to a force model required to be analyzed. From Fig.1, when the end-effector compliantly performs the contourfollowing on the object, the desired force trajectories are usually designed such that the force elements are along the directions normal to the object's surface, whereas the desired position trajectories are along the directions tangent to the object surface. To make such complex problem more tractable, some mild assumptions are given below.

- There is only single contact point.

- The shape of the object is smooth.

- Hook's Law can be applied to the contact force model.

- The friction forces are small and hence can be negligible.

- $x_{c}, \dot{x}_{c}, f_{c}$ and $\dot{f}_{c}$ are measurabe.

Based on the assumptions above, the moment (vector) is approximately zero for the single contact point and the force elements tangential to the object surface are regarded as zero. Therefore, the vector of contact force and contact moment is defined as $F_{c}=$ $\left[f_{c}^{T} m_{c}^{T}\right]^{T}=\left[f_{c}^{T}, 0,0,0\right]^{T}$, where $f_{c}$ and $m_{c}$ represent the force vector and the moment vector, respectively. Futhermore, applying Hook's Law to the contact force model, $f_{c}$ can be found as $f_{c}=-k_{e}\left(x_{p}-x_{s}\right)$, where $x_{s}$ is the end-effector position on the surface of the object before deformation and satisfies that $x_{p}-x_{s}$ is parallel to $f_{c}$.

As mentioned earlier, our objective is to perform hybrid force/position control in the contour-following task. However, as a matter of fact, the precise " desired contour " on the object surface is difficult to be specified in the presence of uncertainty in the object shape. Hence, the desired positional trajectory after priorly described in the world frame as $\widehat{x_{p_{d}}} \in \Re^{3}$ along the undeformed contour on the object surface, needs to be repetitively corrected in a moving frame until the aforementioned objective is met. Therefore, consider a moving frame as $\{V\}$ affixed to $x_{s}$ consisting of three orthonormal bases $n_{1}, n_{2}$, and $n_{3}$ as depicted in Fig.2. The first basis vector $n_{1}$ representing the direction of contact force $f_{c}$ is expressed as

$$
n_{1}=-\frac{f_{c}}{\left\|f_{c}\right\|}
$$




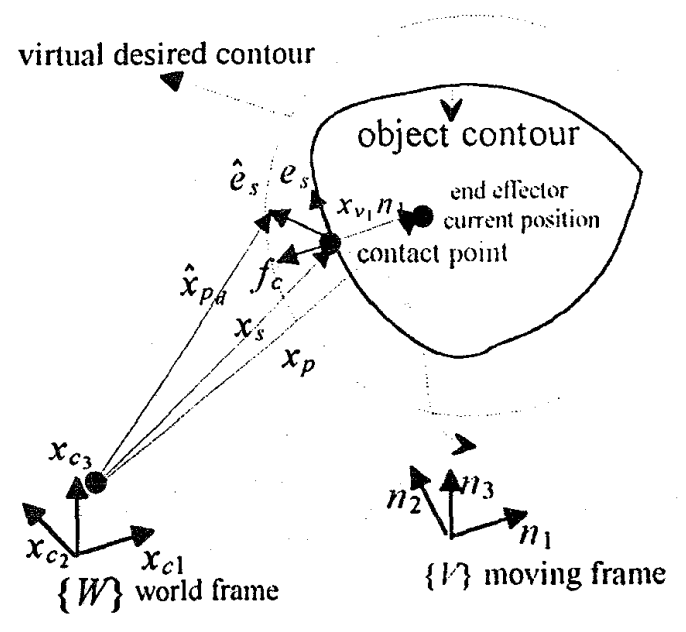

Figure 2: The end effector contacting the object

under assumption that the end-effector is always in contact with the object. Obviously, in the absence of friction force, $n_{1}$ will be normal to the object surface assuming the deformation is rather small. After $n_{1}$ is given, the desired force trajectory $f_{d}$ can then be easily expressed in this moving frame as follows:

$$
f_{d}=f_{d_{m}} n_{1},
$$

where $f_{d_{m}}=\left\|f_{d}\right\|$ is the scalar desired force magnitude. The second basis vector $n_{2}$ representing the direction of the desired positional trajectory direction is constructed via the following procedure. First, let

$$
\widehat{e}_{s}=\widehat{x_{p_{d}}}-x_{s}=\widehat{x_{p_{d}}}-\left(x_{p}+\frac{f_{c}}{k_{e}}\right)
$$

represent the virtual position error between the virtual desired trajectory $\widehat{x_{p_{d}}}$ and the contact point on the undeformed object surface $x_{s}$. Next, we define the trajectory error $e_{s}$ tangential to the object surface as

$$
e_{s}=\widehat{e}_{s}-\left(\widehat{e}_{s} \cdot n_{1}\right) n_{1}=\widehat{e}_{s}-\widehat{e}_{s}^{T} n_{1} n_{1},
$$

where '.' represents the inner product operator. Then, $n_{2}$ is defined as follows:

$$
n_{2}= \begin{cases}\frac{e_{s}}{\left\|e_{e}\right\|,}, & \left\|e_{s}\right\| \neq 0 \\ 0, & \text { otherwise }\end{cases}
$$

Finally, the last basis vector is given as $n_{3}=n_{1} \times n_{2}$, where ' $x$ ' represents the outer product operator.

[Remark]:

In fact, if the direction of friction force is opposite to the direction of the end-effector velocity, then $n_{1}$ can be modified as follows:

$$
n_{1}=-\frac{f_{c}-f_{c}^{T} n_{v} n_{v}}{\left\|f_{c}-f_{c}^{T} n_{v} n_{v}\right\|},
$$

where $n_{v}=\frac{\dot{x}_{p}}{\left\|\dot{x}_{p}\right\|}$.

Practically speaking, the end-effector position expressed in the frame $\{V\}$ will have nonzero value only along $n_{1}$ axis but zero value along the other axes. Let $x_{v 1}$ denote that nonzero scalar value, and thus the practical end-effector position may be written as

$$
x_{p}=x_{s}+x_{v_{1}} n_{1}
$$

Clearly, $x_{s}$ will exactly follow $\widehat{x_{p_{d}}}$ if $\widehat{e_{s}}$ can approach zero. Furthermore, let the desired position trajectory be repetitively updated as follows:

$$
{\widehat{x_{p_{d}}}}^{(k+1)}={\widehat{x_{p_{d}}}}^{(k)}-\left({\widehat{e_{\mathrm{s}}}}^{\left({ }^{k}\right)} \cdot n_{1}{ }^{(k)}\right) n_{1}{ }^{(k)},
$$

where the index $k$ is the number of times for which the end-effector goes around the object.

To derive the contact force model subject to uncertain geometric object surface containing the point, $x_{s}$. So long as $k_{e}$ is precisely known and the desired force trajectory $f_{d}$ is formulated as follows:

$$
f_{d}=k_{e} \frac{f_{d_{m}}}{k_{e}} n_{1}=k_{e} x_{f_{d}} n_{1},
$$

where $x_{f_{d}}=\frac{1}{k_{e}} f_{d_{m}}$ is given, then the force error vector

$$
e_{f}=f_{d}+f_{c}=e_{f_{m}} n_{1}=k_{e}\left(x_{f_{d}}-x_{v_{1}}\right) n_{1}
$$

can be replaced by the the position error vector $e_{p_{f}}=$ $e_{x f} n_{1}$, where $e_{x_{f}}=x_{f_{d}}-x_{v_{1}}$. Define the desired position trajectory in the world frame can be expressed as follows:

$$
\begin{aligned}
x_{p_{d}} & =x_{s}+e_{s}+x_{f_{d}} n_{1} \\
& =x_{p}+\frac{1}{k_{e}} f_{c}+e_{s}+x_{f d} n_{1}
\end{aligned}
$$

Hence, the dynamics (3) can be transformed to a position control problem. In fact, if $x_{p}$ can approach $x_{p_{d}}$, then the contour-following task is solved. When the desired position trajectory $x_{p_{d}}$ is given by equation (12), the desired orientation trajectory is denoted as $x_{m d}$, which is designed from the need of task or from the moment siganls [6]. Let $x_{d}=\left[x_{p_{d}}^{T} x_{m_{d}}^{T}\right]^{T}$ represent the desired position/orientation trajectory. Then, the tracking error $e$ is defined as $e=x_{c}-x_{d}=\left[e_{p}^{T}, e_{m}^{T}\right]^{T}$, where $e_{p}=x_{p_{d}}-x_{p}$ and $e_{m}=x_{m d}-x_{m}$. Now define the sliding mode vector $s=\left[s_{1}, \cdots, s_{6}\right]^{\mathrm{T}}$ as $s=\dot{e}+\lambda e$, where $\lambda$ is a positive constant so that the dynamic equation (3) can be rewritten as follows:

$$
M \dot{s}=M \dot{x}_{r}+C \dot{x}_{c}+D+G-F_{c}-F
$$

Design the control law as

$$
F=K s+C s+h+f_{d}+f_{e}
$$


where $K=\operatorname{diag}\left(k_{1} \ldots, k_{6}\right)$ is a positive definite matrix, $h=\left[h_{1}, \cdots, h_{6}\right]^{T}=M \dot{x}_{r}+C \dot{x}_{c}+G+D$ is the robot dynamics term with $x_{r}=\dot{x}_{d}+\lambda e$, and $f_{e}=\left[\frac{k_{e}}{\lambda} \dot{e}_{p}^{T} n_{1} n_{1}^{T}, 0,0,0\right]^{T}$ is additional compensation vector, then equation (13) can be written as:

$$
M \dot{s}=-K_{e} s-C s,
$$

where $K_{e}$ is expressed as:

$$
K_{e}=K+\left(\begin{array}{cc}
\frac{k_{e}}{\lambda} n_{1} n_{1}^{T} & 0_{3 \times 3} \\
0_{3 \times 3} & 0_{3 \times 3}
\end{array}\right)
$$

In fact, $K_{e}$ is a positive definite matrix and can be derived from the expression of $n_{1} n_{1}^{T} e_{p}=e_{p_{f}}=\frac{1}{k_{e}} e_{f}$. Apparently, if the robot dynamic system is reduced into the equation (15) by the control law (14), then the system tracking error $e$ will be driven to zero asymptotically [12]-[13]. Unfortunately, the prsent problem is much more difficult than those proposed works [8],[10] because $x_{s}, e_{s}$ and $n_{1}$ now involve the system states such as $x_{p}$ and $f_{c}$, and the derivatives of those states are hardly measurable, which hence results in inaccessibility to the signal $\ddot{p_{p}}$.

On the other hand, it is well known that computed torque control algorithm appears to be very complicated in the case of multi-degree-freedom robot manipulators. The sensing time of the force/torque sensor is much longer than that of the motor encoder, measuring the position of robot manipulators. Consequently, to obtain reasonally good performance of the overall controlled system, it is important to maintain high enough control servo rate as well as to take as much much manipulator dynamics into account as possible, the control algorithm to be designed should meet the objectives of complete dynamics control and simple implementation. Given this aim, in the next section we will propose an adaptive fuzzy hybrid force/position control scheme as a solution.

\section{Adaptive Fuzzy Hybrid Force/Position Control}

Referring to section 2, the control law is given as follows:

$$
F=K s+f_{d}+f_{e}+f_{s},
$$

where $f_{s}=\left[f_{s_{1}}, \cdots, \mathrm{f}_{\mathrm{s} 6}\right]^{T}$ represents the fuzzy control compensator to compensate for the nonlinear function vector $h$ consisting of the robot dynamics and the additional uncertainties excited by the contact force. Consider the fuzzy rule structure based on as a decentralized concept, consisting of with $R_{1}, R_{2}, \ldots$, and $R_{6}$

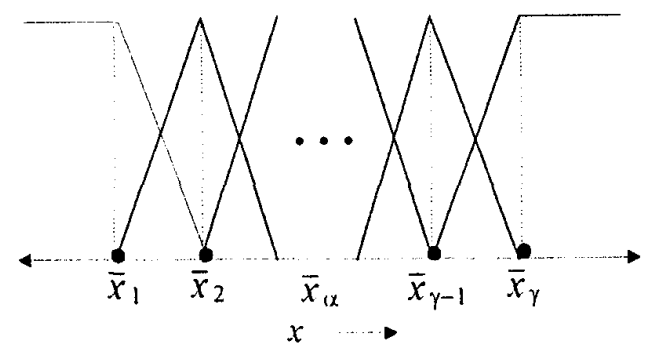

Figure 3: The membership function candidates

rule tables as follows:

$R_{1}[i]:$ If $x_{c 1}$ is $L_{1}^{(i)}$ and $s_{1}$ is $M_{1}^{(i)}$, then $f_{s_{1}}$ is $Q_{1}^{(i)}$
$\quad \vdots$
$R_{6}[i]:$ If $x_{c 6}$ is $L_{6}^{(i)}$ and $s_{6}$ is $M_{6}^{(i)}$, then $f_{s_{6}}$ is $Q_{6}^{(i)}$

where $L_{j}^{(i)}, M_{j}^{(i)}$ and $Q_{j}^{(i)}$ represent the fuzzy sets associated with the $i$-th rule of the $j$-th rule table, corresponding to fuzzy variables $x_{c j}, s_{j}$ and $f_{s_{j}}$, respectively. Based on this rule structure, the sup-min compositional operator and center-of-area defuzzifier strategy are choosen here [10]. Thus the $j$-th element of the fuzzy output vector $f_{s}$ can be derived as follows [10]:

$$
f_{s j}=\Theta_{j}^{T} \xi_{j},
$$

where $\Theta_{j}$ is the $j$-th column vector of an $\Upsilon \times 6$ constant parameter matrix $\Theta$ and $\xi_{j}$ is the $j$-th column vector of an $\Upsilon \times 6$ regressor matrix $\xi$, with $\Upsilon$ being the rule number for every rule table.

Here, our objective is to determine an optimal parameter matrix, with minimal magnitude, $\Theta^{*}=$ $\left[\Theta_{1}^{*} \cdots \Theta_{j}^{*} \cdots \Theta_{6}^{*}\right]$ that can robustify the closed-loop system, and a suitable regressor matrix which can reduce the complex implementation to a simple one. To achieve this goal, the membership functions $\mu\left(x_{c j}\right)$ and $\mu\left(s_{j}\right)$ (see Fig. 3) are set to be:

$$
\mu(x)= \begin{cases}1, & \text { as } x=\bar{x}_{\alpha} \\ 0, & \text { as } x \geq \bar{x}_{\alpha+1} \text { or } x \leq \bar{x}_{\alpha-1} \\ (0,1], & \text { otherwise }\end{cases}
$$

where $\bar{x}_{\alpha}$ is denoted as the center of a fuzzy set with $\alpha$ being any integer index and satisfying $1<\alpha<\gamma$, leading to much shorter computation time and facilitating subsequent determination of the parameter matrix [10]. Before defining the optimal parameters, we 


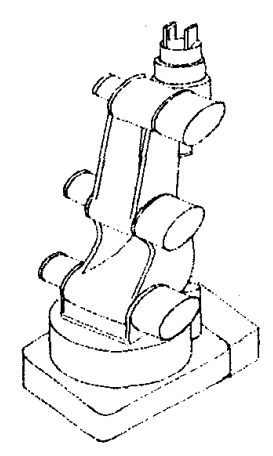

Figure 4: The A-type robot arm

define the modified sliding mode vector $s_{\Delta}$, as follows:

$$
s_{\Delta_{j}}= \begin{cases}s_{j}-\alpha_{j}, & \text { as } s_{j}<\alpha_{j} \\ s_{j}-\beta_{j}, & \text { as } s_{j}>\beta_{j} \\ 0, & \text { othewise }\end{cases}
$$

It is obvious that if $s_{\Delta_{j}} \neq 0$, then $s_{j}$ will be outside a deadzone range $\phi=\left[\alpha_{j}, \beta_{j}\right]$. Based on this fact, then the $j$-th column vector of optimal parameter matrix $\Theta^{*}$ is defiined as follows:

$$
\begin{aligned}
\Theta_{j}^{*}= & \operatorname{argmin}\left[\sup _{x \in E} \Theta_{j}^{T} \xi_{j} \operatorname{sgn}\left(s_{\Delta_{j}}\right)\right. \\
& \left.\geq\left|h_{j}\right|\right], \text { for } s_{\Delta_{j}} \neq 0,
\end{aligned}
$$

where $z=\left[x_{c}^{T}, s^{T}\right]^{T}$ is all input fuzzy variables and $E$ is a compact domain set. Consider the adaptive control law is given as follows:

$$
\dot{\Theta}_{j}=r s_{\Delta_{j}} \xi_{j}, \quad \text { for } z \in E,
$$

where $r$ is a positive constant. Furthermore, assume that $\left|h_{j}\right|$ can be bounded by a smooth function $H_{j}\left(x_{c j}, s_{j}\right)$ when $z$ falls into the compact domain set $E$. Then, the following theorem is valid.

Theorem 1 If the control law and the update law are given as in equation (16) and in equation (22), then the tracking error vector $e$ will converge to a neighborhood of zero asymptotically.

\section{Simulation Results}

A five degree-of-freedom (DOF) articulated robot arm is set up in the Intelligent Robot Laboratory of Dept. of Computer Science \& Information Engineering in National Taiwan University, as depicted in Fig. 4. An elliptical cylinder object is located on the table, expressed as $\frac{x_{c_{1}}{ }^{2}}{50^{2}}+\frac{\left(x_{c_{2}}-300\right)^{2}}{25^{2}}=1$ and $0 \leq x_{\mathfrak{c} 3} \leq 50$. At the beginning, the virtual desired contour is given as a circle of radius $50 \mathrm{~mm}$ in $x_{c 1}-x_{c 2}$

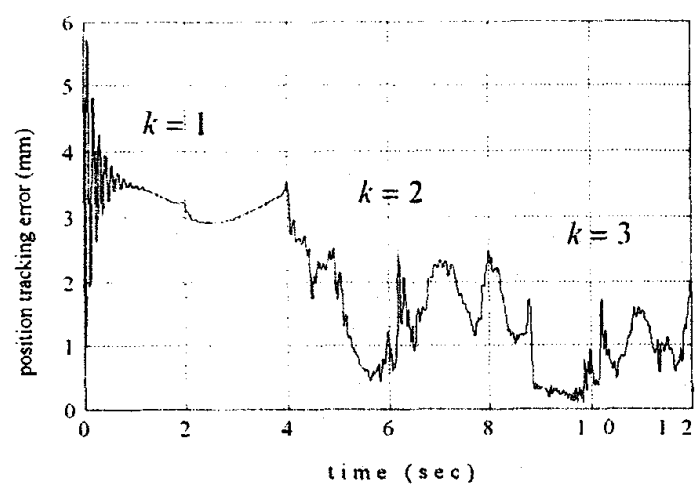

Figure 5: The result of position tracking error $\left\|e_{p}\right\|$

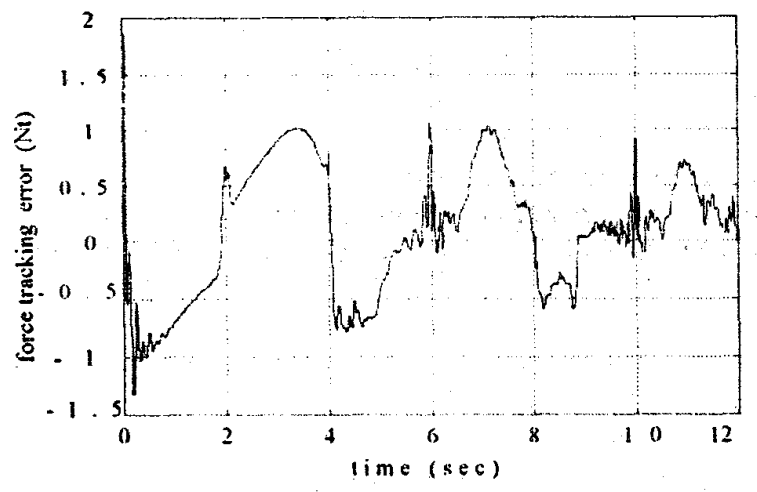

Figure 6: The result of force tracking error $e_{f_{m}}$

plane with the expression: $\frac{x_{c 1}^{2}}{50^{2}}+\frac{\left(x_{c 2}-300\right)^{2}}{50^{2}}=1$ and $x_{c 3}$ is a sinusoidal wave. The desired position trajectories corresponding to this virtual contour are given as $x_{c 1}(t)=50 \cos (0.5 \pi t), x_{c 2}(t)=300+50 \sin (0.5 \pi t)$ and $x_{c 3}(t)=25(1-\cos (0.5 \pi t))$. The desired force magnitude trajectory $f_{d_{m}}$ is given as $f_{d_{m}}=10-5 \exp (-t) \mathrm{Nt}$ with inintial contact force $5 N t$ being given. The total rule number of fuzzy controller is $6 \times 21 \times 21$ and initial parameter matrix of fuzzy rules, $\Theta$, are set to zeros. The stiffness $k_{e}=10(\mathrm{Nt} / \mathrm{mm})$ is given. The norm of position trajectory error, $\left\|e_{p}\right\|$ is listed in Fig. 5. At the beginning, since initial parameter matrix are set to zeros, which is similar to only use the PD controller to compensate for the uncertainties in the first period of tracking motion, after the first period the tracking error is quickly driven toward zero. On the other hand, the force error $e_{f_{m}}$ is given in the Fig. 6, we can find that the error is converging to zero. Besides, the virtual desired position trajectory $\widehat{x_{p}}$ converges to the real contour of the object, shown in Fig.7. 


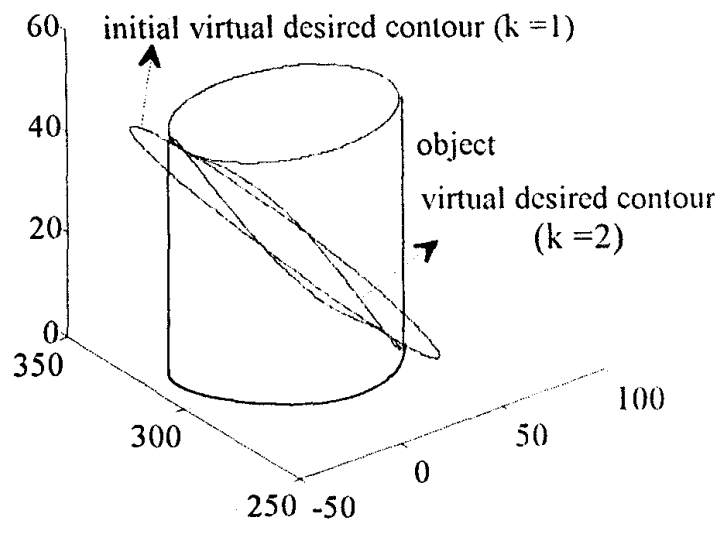

Figure 7: The result of virtual desired trajectory $\widehat{x_{p_{d}}}$

\section{Conclusions}

We had proposed an adaptive fuzzy hybrid force/position controller, which can update fuzzy rules to compensate for the robot dynamics along with the force dynamics induced by the contact between endeffector and object, and identify the actual desired contour of the object. For illustration, a simulation is conducted and satisfactory results have been observed. Actual experiment is under way.

\section{References}

[1] M. Blauer and P. R. Belanger, "State And Parameter Estimation for Robotic Manipulators Using Force Measurements, " IEEE Trans. Autom. Cont., vo. 32, Dec. 1987

[2] T. Yoshikawa and A. Sudou, "Dynamic Hybrid Poition/Force Control of Robot Manipulators-OnLine Estimation of Unknown Constraint, " IEEE Transactions on Robotics and Automation vo. 9, no. 2, pp. 220-225, April 1993

[3] J. -H. Jean and L.-C. Fu, " An Adaptive Control Scheme for Coordinated Multimanipulator Systems, "IEEE Transactions on Robotics and Automation vo. 9, no. 2, pp. 226-231, April. 1993

[4] S. A. Schneider and Robert H. Cannon, Jr, "Object Impedance Control for Cooperative Manipulation: Theory and Experimental Results, "IEEE
Transactions on Robotics and Automation vo. 8, no. 3, pp. 383-394, June 1992

[5] W. McCormick and H. M. Schwartz, "An Investigation of Impedance Control for Robot Manipulators," The International Journal of Robotics Research vol. 12, no. 5, October 1993, pp. 473-489

[6] K. Guglielmo and N. Sadegh, "Implementating a Hybrid Learning Force Control Scheme, "IEEE Control Systems vo. 14, no. 1, pp. 72-79 Feb. 1994

[7] D. Jeon and M. Tomizuka, "Learning Hybrid Force and Position Control of Robot Manipulators," IEEE Transactions on Robotics and Automation vo. 9, no. 4, pp. 423-432, Aug. 1993

[8] M. H. Raibert and J. J. Craig, "Hybrid Position/Force Control of Manipulators" ASME Jornal of Dynamic Systems, Measurement, and Control vol. 102 pp. 126-133, 1981

[9] F. -Y. Hsu and L. -C. Fu, "Adaptive Robust Fuzzy Control for Robot Manipulators "IEEE Conference on Robotics and Automation, pp. 629-634, 1994

[10] F. -Y. Hsu and L. -C. Fu, "A New Design of Adaptive Robust Fuzzy Controller for Robot Manipulators, "IEEE Conference on Robotics and Automation, pp. 863-868, 1995

[11] J. Marsden, Elementary Classical Analysis. San Franciso: W. H. Freeman, 1974

[12] J-J E. Slotine and W. Li, Applied Nonlinear Control, Englewood Cliffs, NJ: Prentice Hall, pp. 278$284,1991$.

[13] G. Niemeyer, J-J E. Slotine, " Performance in Adaptive Manipulator Control ", The International Journal of Robotics Research, vol. 10, no. 2, pp. 149-161, 1991. 